\title{
Thermodynamics of thallium alkanoates III. Heat capacity and thermodynamic functions of thallium(I) n-tetradecanoate from 7 to $450 \mathrm{~K}^{a}$
}

\author{
S. P. NGEYI, ${ }^{b}$ E. F. WESTRUM, Jr., \\ Department of Chemistry, University of Michigan, \\ Ann Arbor, Michigan 48109, U.S.A. \\ F. L. LOPEZ DE LA FUENTE, J. A. R. CHEDA, and \\ Departamento de Quimica Fisica, Facultad de Ciencias Quimicas, \\ Universidad Complutense, 28040 Madrid, Spain \\ F. FERNANDEZ-MARTIN \\ Instituto del Frio, CSIC, 28040 Madrid, Spain
}

(Received 20 March 1986; in final form 9 July 1986)

\begin{abstract}
The heat capacity of thallium(I) $n$-tetradecanoate was measured by adiabatic calorimetry to $350 \mathrm{~K}$ and by d.s.c. from 235 through $460 \mathrm{~K}$. Good agreement between the methods was obtained within the common temperature range. Several phases were observed in the sample. Two solid-to-solid transitions were found, a very sharp one at $318.5 \mathrm{~K}\left(C_{p, \mathrm{~m}} / R\right.$ for the maximum is about $10^{4}$; enthalpy $\left(\Delta_{\mathrm{trs}} H_{\mathrm{m}} / R\right)$ and entropy $\left(\Delta_{\mathrm{trs}} S_{\mathrm{m}} / R\right)$ increments are $1913.5 \mathrm{~K}$ and 6.00, respectively) and the other at $378.0 \mathrm{~K}: \Delta_{\mathrm{trs}} H_{\mathrm{m}} / R=346 \mathrm{~K}, \Delta_{\mathrm{trs}} S_{\mathrm{m}} / R=0.92$. Between these transitions there is a broad diffuse hump whose maximum is at about $360 \mathrm{~K}$. The sample melts into a liquid-crystal phase at $396.3 \mathrm{~K}: \Delta_{\mathrm{trs}} H_{\mathrm{m}} / R=754 \mathrm{~K}, \Delta_{\mathrm{trs}} S_{\mathrm{m}} / R=1.9$. Finally, "clearing" occurs at $460.7 \mathrm{~K}: \Delta_{\mathrm{trs}} H_{\mathrm{m}} / R=201 \mathrm{~K}, \Delta_{\mathrm{trs}} S_{\mathrm{m}} / R=0.44$. Smoothed thermophysical properties are tabulated through "clearing".
\end{abstract}

\section{Introduction}

The thermophysical properties of thallium(I) n-hexanoate and thallium(I) $n$-heptanoate have been measured earlier ${ }^{(1,2)}$ and properties of these salts were established. Below ambient temperatures thallium(I) $n$-hexanoate, showed two solid-to-solid transitions ( 203.5 and $280.3 \mathrm{~K}$ ) while thallium(I) $n$-heptanoate showed up to five transitions, one of which, however, is presumably due to the formation of a (salt + acid) complex.

This paper deals with the study of thallium $n$-tetradecanoate. Two techniques were used: d.s.c. from 235 to $450 \mathrm{~K}$ and sub-ambient adiabatic calorimetry from 7

${ }^{a}$ The previous papers in this series are references 1 and 2 .

${ }^{b}$ Present address: c/o Mr P. N. Limen, Posts and Telecommunications Delegation, Mankon Town, Bamenda-Mezam Division, North West Province, Republic of Cameroon. 
to $350 \mathrm{~K}$ to delineate the morphology of the heat capacity from $7 \mathrm{~K}$ through melting to "clearing" and hence provide the energetic spectrum of the material. Several phases were found including a mesomorphic liquid crystal. The phase transitions can be summarized as:

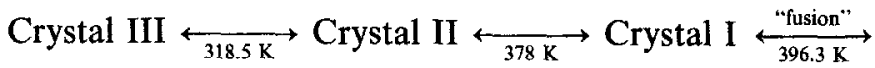

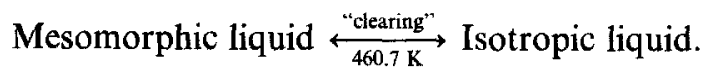

\section{Experimental}

The sample was prepared by reaction of $n$-tetradecanoic acid with thallium carbonate in dry methanol, according to the procedure of Meisel et al. ${ }^{(3)} \mathrm{A}$ d.s.c. purity determination using fractional-fusion techniques indicated 99.85 moles per cent of liquid-soluble solid-insoluble purity.

Adiabatic calorimetry. The programming, data logging, and calorimetry were done by computerization described elsewhere. ${ }^{(4,5)}$ Measurement of mass, time, resistance, and weight corrections were referred to calibrations of the National Bureau of Standards.

The sample was loaded into the gold-plated copper calorimeter designated W-62. After evacuation, dry helium gas was added at a pressure of $0.3 \mathrm{kPa}$ to facilitate thermal equilibrium during data acquisition. The sealed calorimeter was then placed in the Mark X cryostat and assembled for data acquisition. The sample mass was $26.844 \mathrm{~g}(\hat{=} 0.062176 \mathrm{~mol})$.

Buoyancy corrections were calculated using a density of $2.2 \mathrm{~g} \cdot \mathrm{cm}^{-3}$, a quantity evaluated from the densities of the earlier members studied. ${ }^{(6)}$ Crystallographic information on the density of none of the neighboring members is available; thallium acetate has a density of $3.6 \mathrm{~g} \cdot \mathrm{cm}^{-3}$.

The thermal history of the thallium $n$-tetradecanoate sample is shown below, where solid arrows indicate cooling and dashed arrows indicate data acquisition ranges for various series.

$$
\begin{aligned}
& 298 \mathrm{~K} \stackrel{2 \mathrm{~h}}{\longrightarrow} 240 \mathrm{~K} \underset{\text { Series } \mathrm{I}}{\stackrel{4 \mathrm{~h}}{\longrightarrow}} 301 \mathrm{~K} \stackrel{13 \mathrm{~h}}{\longrightarrow} 117 \mathrm{~K} \stackrel{11 \mathrm{~h}}{\longrightarrow} 256 \mathrm{~K} \stackrel{14.5 \mathrm{~h}}{\longrightarrow} \\
& 57 \mathrm{~K} \underset{\text { Series III }}{\stackrel{0.5 \mathrm{~h}}{\rightarrow}} 133 \mathrm{~K} \stackrel{46 \mathrm{~h}}{\longrightarrow} 6.7 \mathrm{~K} \underset{\text { Series IV }}{\stackrel{2 \mathrm{~h}}{\longrightarrow}} 13.6 \mathrm{~K} \stackrel{0.5 \mathrm{~h}}{\longrightarrow} 6.9 \mathrm{~K} \underset{\text { Series } \overrightarrow{\mathrm{V}}}{\stackrel{19 \mathrm{~h}}{\longrightarrow}} \\
& 64.7 \mathrm{~K} \stackrel{31 \mathrm{~h}}{\longrightarrow} 60 \mathrm{~K} \underset{\text { Series } \overrightarrow{\mathrm{vI}}}{\stackrel{11 \mathrm{~h}}{\longrightarrow}} 342 \mathrm{~K} \stackrel{12.5 \mathrm{~h}}{\longrightarrow} 256 \mathrm{~K} \underset{\text { Series } \overrightarrow{\mathrm{v} 11}}{\stackrel{7 \mathrm{~h}}{\longrightarrow}} 348 \mathrm{~K} \stackrel{17 \mathrm{~h}}{\longrightarrow} \\
& 232 \mathrm{~K} \underset{\text { Series }}{\stackrel{8 \mathrm{~h}}{\longrightarrow}} 330 \mathrm{~K} \stackrel{15.5 \mathrm{~h}}{\longrightarrow} 237 \mathrm{~K} \underset{\text { Series } \mathrm{IX}}{\stackrel{4 \mathrm{~h}}{\longrightarrow}} 339 \mathrm{~K} \text {. }
\end{aligned}
$$

Differential scanning calorimetry. A Perkin-Elmer DSC-2 provided with an Intracooler II device was used. Its temperature display was calibrated at the melting temperatures of several high-purity ( $>99.9$ moles per cent) substances ( $n$-undecane, $n$-tridecane, $n$-pentadecane, gallium, stearic acid, benzoic acid, indium, and tin). The 
enthalpies of transitions were determined with high-purity indium (>99.999 moles per cent pure) as reference. For the heat-capacity determination synthetic sapphire was used as an external standard.

\section{Results and discussion}

The experimental heat capacities are given in table 1 for adiabatic calorimetry and in table 2 for d.s.c. and are plotted together in figure 1 for the whole temperature region studied. An expanded-range plot is shown in figure 2 to enhance the features of the $318.5 \mathrm{~K}$ transition. The value of $318.5 \mathrm{~K}$ for the transition obtained by d.s.c. is in good agreement with the value obtained by adiabatic calorimetry. The standard deviations in the adiabatic-calorimetry heat capacities begin at 5 per cent at $7 \mathrm{~K}$ and decrease to 0.3 per cent at $20 \mathrm{~K}$ and to less than 0.1 per cent above $50 \mathrm{~K}$. The standard deviations for the d.s.c. heat capacities are about 3 per cent.

The heat capacities were fitted to a series of orthogonal polynomials in regions of normal heat capacity. Integration of the polynomials yielded the thermodynamic

TABLE 1. Molar heat capacities of thallium(I) $n$-tetradecanoate $\mathrm{C}_{13} \mathrm{H}_{27} \mathrm{CO}_{2} \mathrm{Tl}$ $\left(R=8.3144 \mathrm{~J} \cdot \mathrm{K}^{-1} \cdot \mathrm{mol}^{-1}\right)$

\begin{tabular}{|c|c|c|c|c|c|c|c|c|c|}
\hline$T / \mathbf{K}$ & $C_{p, \mathbf{m}} / R$ & $T / \mathrm{K}$ & $C_{p, \mathrm{~m}} / R$ & $T / \mathbf{K}$ & $C_{p, \mathrm{~m}} / R$ & $T / \mathbf{K}$ & $C_{p, m} / R$ & $T / \mathbf{K}$ & $C_{p, \mathrm{~m}} / R$ \\
\hline \multicolumn{2}{|c|}{ Series I } & 221.31 & 37.017 & 11.40 & 1.339 & 40.26 & 9.991 & \multicolumn{2}{|c|}{ Series VII } \\
\hline 244.15 & 40.205 & 227.66 & 37.832 & 12.29 & 1.497 & 43.42 & 10.927 & $\Delta H_{\mathrm{m}}$ & Detn. H \\
\hline 251.12 & 41.576 & 234.01 & 38.813 & 13.19 & 1.737 & 46.58 & 11.856 & $\Delta H_{\mathrm{m}}^{\mathrm{m}}$ & Detn. I \\
\hline 257.88 & 42.604 & 240.36 & 39.676 & \multirow{2}{*}{\multicolumn{2}{|c|}{ Series V }} & 50.22 & 12.884 & 303.92 & 55.679 \\
\hline 264.64 & 43.812 & 246.71 & 40.755 & & & 54.32 & 13.925 & 308.92 & 57.650 \\
\hline 271.38 & 45.257 & 253.05 & 41.826 & 7.36 & 0.45 & 58.45 & 14.997 & 315.07 & 284.07 \\
\hline 278.11 & 46.761 & \multirow{2}{*}{\multicolumn{2}{|c|}{ Series III }} & 8.07 & 0.63 & 62.64 & 15.996 & 328.09 & 89.862 \\
\hline 284.83 & 48.415 & & & 8.87 & 0.77 & \multirow{2}{*}{\multicolumn{2}{|c|}{ Series VI }} & 340.31 & 81.627 \\
\hline 291.54 & 50.367 & 60.73 & 15.527 & 9.71 & 0.95 & & & 345.63 & 84.316 \\
\hline 298.23 & 52.644 & 65.51 & 16.657 & 10.57 & 1.126 & 73.27 & 18.211 & \multirow{2}{*}{\multicolumn{2}{|c|}{ Series VIII }} \\
\hline \multirow{2}{*}{\multicolumn{2}{|c|}{ Series II }} & 69.55 & 17.486 & 11.45 & 1.300 & $\Delta H_{\mathrm{m}}$ & Detn. A & & \\
\hline & & 73.66 & 18.275 & 12.33 & 1.543 & $\Delta H_{\mathrm{m}}^{\mathrm{m}}$ & Detn. B & $\Delta H_{\mathrm{m}}$ & Detn. J \\
\hline 121.59 & 25.567 & 78.27 & 19.199 & 13.23 & 1.735 & 121.81 & 25.577 & 294.30 & 51.879 \\
\hline 127.25 & 26.179 & 83.36 & 20.215 & 14.14 & 1.994 & $\Delta H_{\mathrm{m}}$ & Detn. C & 314.00 & 60.839 \\
\hline 132.53 & 26.790 & 88.48 & 21.132 & 15.05 & 2.251 & $\Delta H_{\mathrm{m}}$ & Detn. D & 317.65 & 108.49 \\
\hline 137.82 & 27.390 & 93.63 & 21.909 & 15.97 & 2.500 & $\Delta H_{\mathrm{m}}$ & Detn. E & 318.31 & 1086.08 \\
\hline 143.12 & 27.938 & 98.82 & 22.601 & 17.11 & 2.820 & $\Delta H_{\mathrm{m}}$ & Detn. F & 318.42 & 1536.19 \\
\hline 148.43 & 28.576 & 104.03 & 23.344 & 18.47 & 3.213 & 294.21 & 51.280 & 318.48 & 1827.35 \\
\hline 153.74 & 29.119 & 109.26 & 24.001 & 19.82 & 3.626 & 300.14 & 53.312 & 318.50 & 8282.2 \\
\hline 159.06 & 29.686 & 114.50 & 24.694 & 21.19 & 4.049 & $\Delta H_{\mathrm{m}}$ & Detn. $G$ & 318.51 & 3047.6 \\
\hline 164.39 & 30.273 & 119.76 & 25.368 & 22.57 & 4,458 & 312.24 & 59.269 & 318.56 & 1548.7 \\
\hline 169.72 & 30.787 & 125.02 & 26.002 & 23.96 & 4.893 & 317.03 & 139.403 & 318.62 & 2361.8 \\
\hline 175.05 & 31.368 & 130.14 & 26.582 & 25.36 & 5.332 & 318.80 & 2549.48 & 318.62 & 2932.3 \\
\hline 180.38 & 31.980 & \multirow{2}{*}{\multicolumn{2}{|c|}{ Scrics IV }} & 26.77 & 5.770 & 319.02 & 1723.33 & 319.54 & 292.23 \\
\hline 185.71 & 32.568 & & & 28.50 & 6.315 & 319.94 & 309.73 & 323.01 & 79.360 \\
\hline 191.05 & 33.227 & 7.24 & 0.45 & 30.53 & 6.961 & 323.24 & 80.917 & 328.15 & 78.431 \\
\hline 196.39 & 33.844 & 8.05 & 0.62 & 32.57 & 7.611 & 328.34 & 78.579 & \multicolumn{2}{|c|}{ Series IX } \\
\hline 202.24 & 34.544 & 8.86 & 0.75 & 34.62 & 8.244 & 333.49 & 79.034 & 310.78 & 58.816 \\
\hline 208.60 & 35.340 & 9.67 & 0.94 & 36.26 & 8.781 & & & 324.47 & 161.47 \\
\hline 214.95 & 36.098 & 10.53 & 1.104 & 37.76 & 9.259 & & & 336.83 & 79.957 \\
\hline
\end{tabular}


TABLE 2 . Heat capacity of thallium(I) n-tetradecanoate by d.s.c. $\left(R=8.3144 \mathrm{~J} \cdot \mathrm{K}^{-1} \cdot \mathrm{mol}^{-1}\right)$

\begin{tabular}{cccccccccc}
\hline$T / \mathrm{K}$ & $C_{p, \mathrm{~m}} / R$ & $T / \mathrm{K}$ & $C_{p, \mathrm{~m}} / R$ & $T / \mathrm{K}$ & $C_{p, \mathrm{~m}} / R$ & $T / \mathrm{K}$ & $C_{p, \mathrm{~m}} / R$ & $T / \mathrm{K}$ & $C_{p, \mathrm{~m}} / R$ \\
\hline 235 & 38.5 & 280 & 47.7 & \multicolumn{2}{c}{ Trs: (III) $\rightarrow(\mathrm{II})$} & Trs: $(\mathrm{II}) \rightarrow(\mathrm{I})$ & 420 & 82.3 \\
240 & 39.4 & 285 & 49.2 & 330 & 77.3 & 380 & 62.9 & 425 & 82.5 \\
245 & 40.7 & 290 & 50.6 & 335 & 79.2 & 385 & 64.6 & 430 & 82.7 \\
250 & 41.6 & 295 & 55.2 & 340 & 81.0 & 390 & 67.6 & 435 & 82.9 \\
255 & 42.5 & 300 & 54.3 & 345 & 83.4 & Fusion:(I) $\rightarrow(\mathrm{M})$ & 440 & 83.1 \\
260 & 43.5 & 305 & 56.5 & 350 & 86.1 & 405 & 83.5 \\
265 & 44.5 & 310 & 58.5 & 355 & 87.8 & 405 & 80.95 & 450 & 83.8 \\
270 & 45.6 & & & 360 & 86.2 & 410 & 81.4 & & \\
275 & 46.3 & & & 365 & 82.6 & 415 & 81.9 & & \\
& & & 370 & 80.0 & & & & \\
\hline
\end{tabular}

functions. In the region of transitions, excess enthalpies and entropies were obtained by drawing the appropriate lattice curves and assuming isothermal transition. One first-order transition was observed in the heat-capacity curve from the adiabatic calorimetry at $318.5 \mathrm{~K}$. The reproducibility of the heat-capacity curve in this transitional region in our measurements was within the experimental error, as shown in table 3 for several determinations through this transition region. Moreover, enthalpy-type determinations in this normal heat-capacity region were compared with the values obtained from heat-capacity values and the results are shown in table 4.

In table 5 the transition properties obtained by d.s.c. measurements are summarized. The values of the enthalpy and entropy for the transition measured by both techniques are in good agreement.

At clearing an exothermic effect was observed attributable to the decomposition of the sample. For this reason, d.s.c. heat-capacity values could not be obtained in this region.

Lindau et al. ${ }^{(7)}$ observed two transitions below $350 \mathrm{~K}$, viz. $315.2 \mathrm{~K}$ and $309.7 \mathrm{~K}$ giving a total enthalpy of transition of $1323.01 R \cdot \mathrm{K}$. This value is considerably smaller than the $1913.53 R \cdot \mathrm{K}$ value observed in this study. Lindau does not mention the transition at $309.7 \mathrm{~K}$ in a later publication ${ }^{(8)}$ which makes his results in reference (7) above questionable. It is apparent from cooling curves that undercooling occurs in this sample and that the transition to a lower-temperature phase begins to take place only at about $315 \mathrm{~K}, 3 \mathrm{~K}$ below the observed transition temperature.

On the other hand, the d.s.c. cooling thermograms of the (Crystal II-toCrystal III) transition show three peaks that change depending on the thermal treatment of the sample; however, during heating a single peak was always observed.

The smoothed table of thermophysical properties obtained in this work is summarized in table 6 at selected round temperatures. A summary of the observed transition temperatures and literature values for comparison is shown in table 7 .

Utilizing the approach already developed ${ }^{(9-11)}$ for estimating the lattice heat capacity by incrementation with $\mathrm{CH}_{2}$-units for the tetradccanoate referred to 


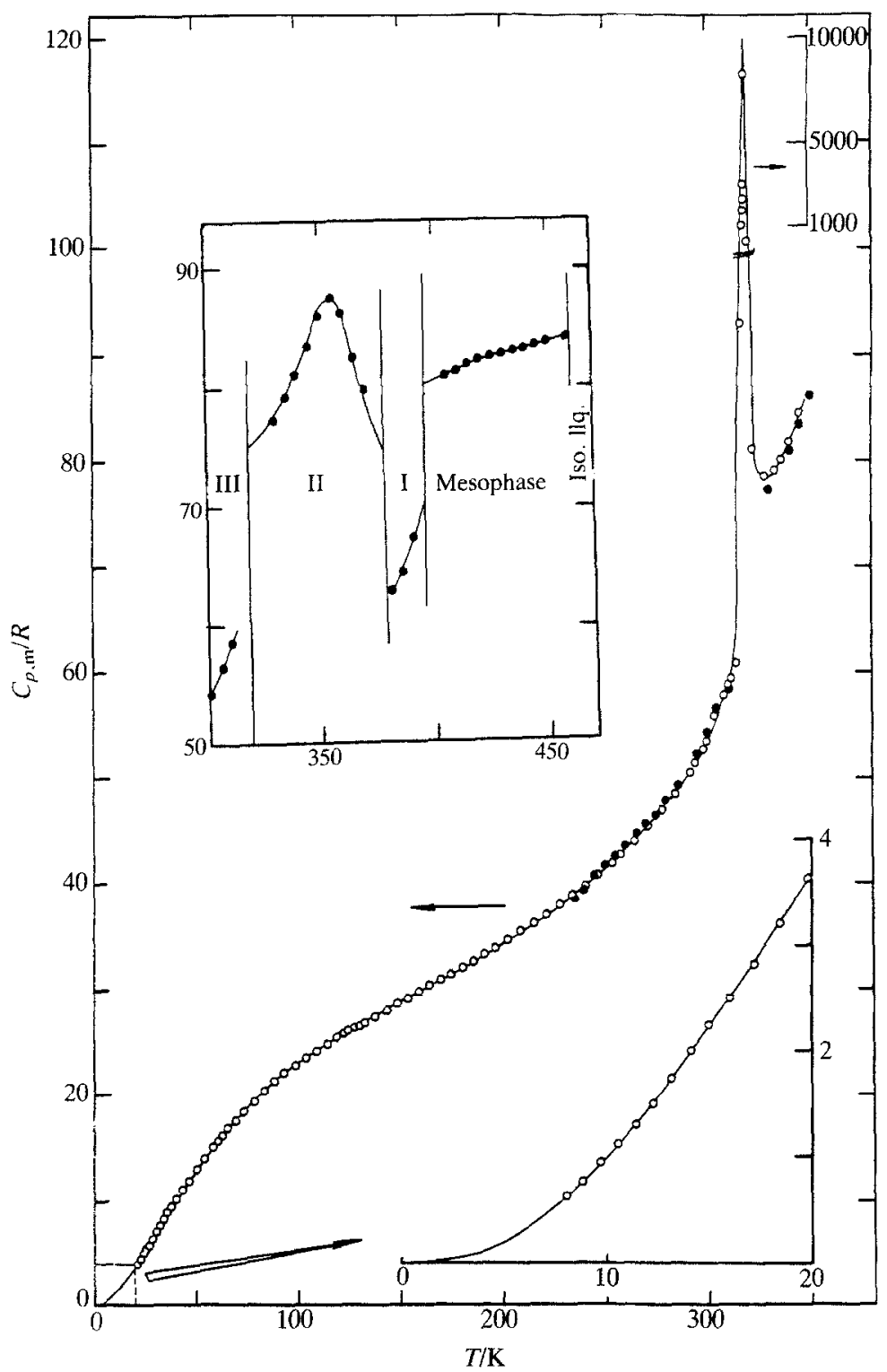

FIGURE 1. Heat-capacity curve for thallium(I) $n$-tetradecanoate. $O$, Adiabatic calorimetric determinations; 0 , d.s.c. determinations.

thallium heptanoate ${ }^{(1)}$ we found that the estimated lattice heat capacity fits within 5 per cent to $230 \mathrm{~K}$ at which temperature a transition in the reference compound commences. Over the region extending up to the first transition in the thallium hexanoate ${ }^{(2)}$ this alternative reference compound provides almost as good a fit even 


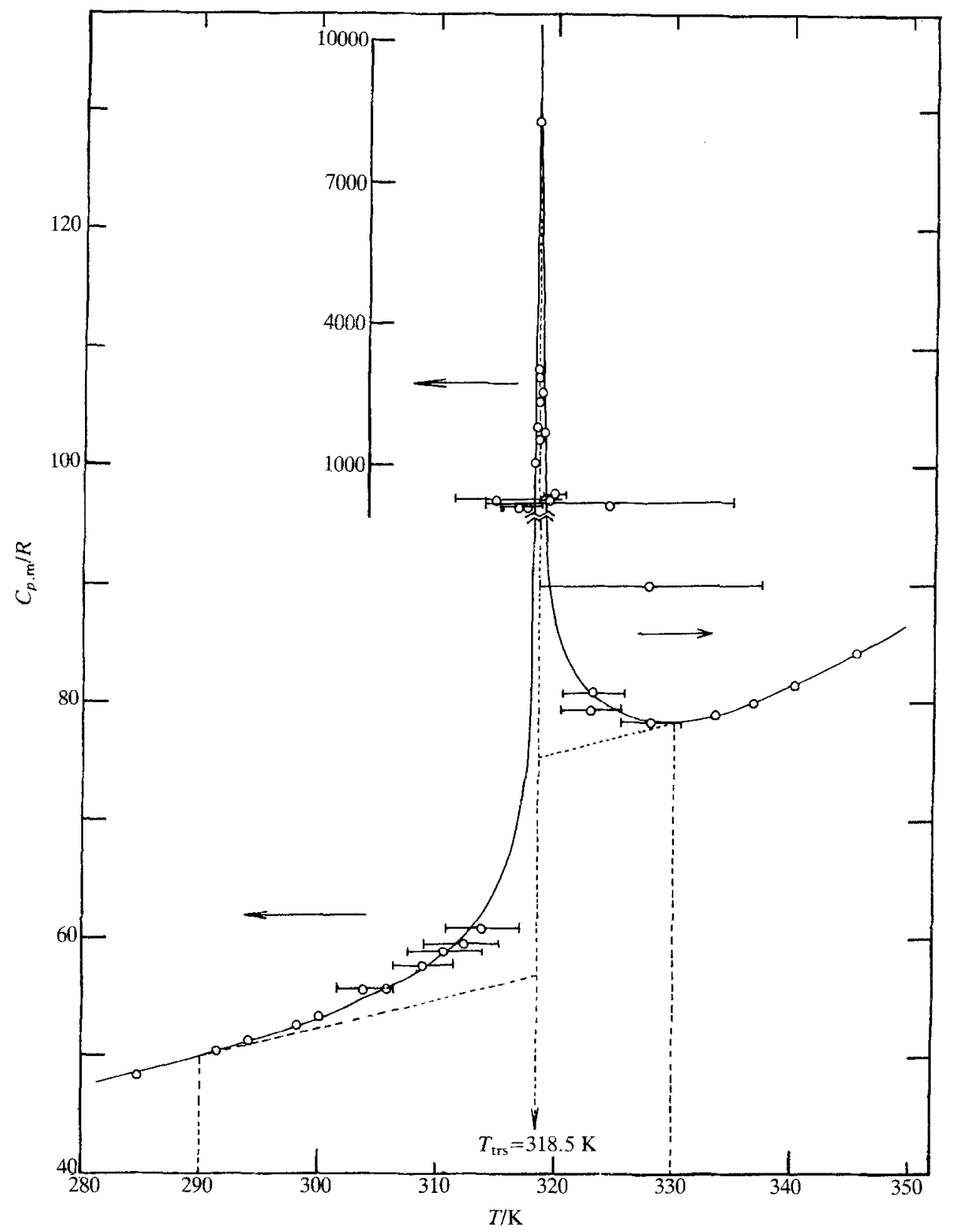

FIGURE 2. Expanded heat-capacity curve of thallium(I) $n$-tetradecanoate in the transition region. 
TABLE 3. Enthalpy and entropy of $318.15 \mathrm{~K}$ transition in thallium(I) $n$-tetradecanoate $\left(R=8.3144 \mathrm{~J} \cdot \mathrm{K}^{-1} \cdot \mathrm{mol}^{-1}\right)$

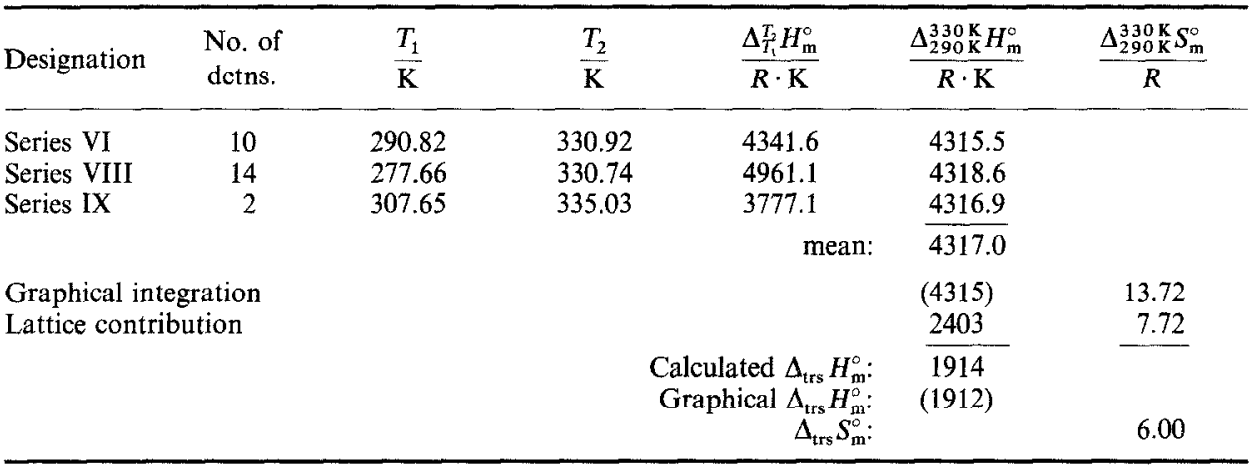

TABLE 4. Comparison of enthalpy-type determinations with integrated heat capacities over the same heat-capacity regions

\begin{tabular}{cccccc}
\hline Designation & $\frac{T_{1}}{\mathrm{~K}}$ & $\frac{T_{2}}{\mathrm{~K}}$ & $\frac{\Delta_{T_{1}}^{T_{2}} H_{\mathrm{m}}}{R \cdot \mathrm{K}}$ & $\frac{\int C_{p, \mathrm{~m}} \mathrm{~d} T}{R \cdot \mathrm{K}}$ & \multirow{2}{*}{$10^{2} \frac{\Delta H_{\mathrm{m}}-\int C_{p, \mathrm{~m}} \mathrm{~d} T}{\Delta H_{\mathrm{m}}}$} \\
\hline $\mathrm{A}$ & 75.05 & 109.12 & 728.04 & 731.65 & 0.12 \\
$\mathrm{~B}$ & 109.11 & 120.80 & 289.35 & 289.22 & 0.05 \\
$\mathrm{C}$ & 122.81 & 165.65 & 1198.82 & 1200.43 & 0.03 \\
$\mathrm{D}$ & 165.62 & 206.96 & 1351.32 & 1355.22 & 0.07 \\
$\mathrm{E}$ & 206.95 & 249.21 & 1602.71 & 1607.60 & 0.07 \\
$\mathrm{~F}$ & 249.20 & 290.82 & 1879.71 & 1881.75 & 0.03 \\
$\mathrm{G}$ & 302.68 & 309.14 & 360.29 & 361.60 & 0.09 \\
$\mathrm{H}$ & 253.82 & 272.53 & 820.33 & 816.09 & 0.12 \\
$\mathrm{I}$ & 272.53 & 301.53 & 1435.62 & 1432.39 & 0.06 \\
$\mathrm{~J}$ & 236.10 & 277.67 & 1766.93 & 1770.63 & 0.05 \\
\hline
\end{tabular}

TABLE 5. Transition properties of thallium(I) $n$-tetradecanoate by d.s.c. (mean values of four determinations)

\begin{tabular}{lccc}
\hline \multicolumn{1}{c}{ Transition } & $\frac{T}{\mathrm{~K}}$ & $\frac{\Delta_{\mathrm{trs}} H_{\mathrm{m}}^{\circ}}{R \cdot \mathrm{K}}$ & $\frac{\Delta_{\mathrm{trs}} S_{\mathrm{m}}^{\circ}}{R}$ \\
\hline Crystal III $\rightarrow$ Crystal II & 318.2 & 1816 & 5.7 \\
Crystal II $\rightarrow$ Crystal I & 378.0 & 346 & 0.92 \\
Crystal I $\rightarrow$ Mesophase & 396.3 & 754 & 1.9 \\
Mesophase $\rightarrow$ Isotropic liquid & 460.7 & 201 & 0.44 \\
\hline
\end{tabular}


TABLE 6. Smooth thermodynamic values at selected temperatures for thallium(I) $n$-tetradecanoate

$$
\left\{R=8.3144 \mathrm{~J} \cdot \mathrm{K}^{-1} \cdot \mathrm{mol}^{-1} ; \Phi_{\mathrm{m}}^{\circ}(T, 0) \stackrel{\text { def }}{=}-\Delta_{0}^{T} H_{\mathrm{m}}^{\circ}(T) / T+\Delta_{0}^{T} S_{\mathrm{m}}^{\circ}(T)\right\}
$$

\begin{tabular}{|c|c|c|c|c|c|c|c|c|c|}
\hline$\frac{T}{\mathrm{~K}}$ & $\frac{C_{\mathrm{p}, \mathrm{m}}}{R}$ & $\frac{\Delta_{0}^{T} S_{\mathrm{m}}^{\circ}(T)}{R}$ & $\frac{\Delta_{0}^{T} H_{\mathrm{m}}^{\circ}(T)}{R \cdot \mathbf{K}}$ & $\frac{\Phi_{\mathrm{m}}^{\circ}(T, 0)}{R}$ & $\frac{T}{\mathrm{~K}}$ & $\frac{C_{p, \mathrm{~m}}}{R}$ & $\frac{\Delta_{0}^{T} S_{\mathrm{m}}^{\circ}(T)}{R}$ & $\frac{\Delta_{\mathrm{v}}^{T} H_{\mathrm{m}}^{\circ}(T)}{R \cdot \mathrm{K}}$ & $\frac{\Phi_{\mathrm{m}}^{\circ}(T, 0)}{R}$ \\
\hline & & Phase III & & & 325 & 78.70 & 68.11 & 11672.4 & 35.71 \\
\hline 0 & 0 & 0 & 0 & 0 & & [76.90] & {$[62.11]$} & [9758.8] & [29.84] \\
\hline 10 & 0.998 & 0.333 & 2.496 & $\begin{array}{ll}6 & 0.083\end{array}$ & $330^{\circ}$ & 78.2 & & 12060.0 & 36.34 \\
\hline 15 & 2.225 & 0.955 & 10.378 & $\begin{array}{ll}3 & 0.263\end{array}$ & & [78.2] & {$[63.45]$} & [10146.5] & {$[30.55]$} \\
\hline 20 & 3.678 & 1.792 & 25.102 & $2 \quad 0.537$ & 340 & 81.5 & 71.81 & 12856.8 & 34.00 \\
\hline 25 & 5.218 & 2.777 & 47.311 & 0.885 & & & {$[65.81]$} & [10943.2] & [33.62] \\
\hline 30 & 6.788 & 3.867 & 77.32 & 1.290 & 350 & 86.1 & 74.24 & 13697 & 35.11 \\
\hline 35 & 8.382 & 5.033 & 115.24 & 1.740 & & & {$[68.24]$} & [11784] & {$[34.57]$} \\
\hline 40 & 9.921 & 6.254 & 161.05 & 2.228 & 360 & 86.2 & 76.72 & 14570 & 36.25 \\
\hline 45 & 11.395 & 7.507 & 214.33 & 2.745 & & & [70.72] & [12656] & {$[35.56]$} \\
\hline 50 & 12.809 & 8.782 & 274.89 & 3.285 & 370 & 80.0 & 79.00 & 15400 & 37.38 \\
\hline 60 & 15.35 & 11.347 & 415.95 & 4.415 & & & {$[73.00]$} & [13487] & [36.55] \\
\hline 70 & 17.60 & 13.886 & 580.9 & 5.587 & $378.0^{b}$ & $(>400)$ & $(80.65)$ & (16016) & $(38.28)$ \\
\hline 80 & 19.57 & 16.37 & 767.0 & 6.780 & & 74.23 & [74.65] & [14102] & [37.34] \\
\hline 90 & 21.30 & 18.77 & 971.5 & 7.980 & & & Phase I & & \\
\hline 100 & 22.82 & 21.10 & 1192.3 & 9.176 & & & Fnase I & & \\
\hline 120 & 25.38 & 25.49 & 1675.1 & 11.534 & $378.0^{b}$ & $(>400)$ & $(81.57)$ & (16364) & $(38.28)$ \\
\hline 140 & 27.63 & 29.58 & 2205.4 & 13.823 & & [63.1] & {$[74.65]$} & [14102] & {$[37.34]$} \\
\hline 160 & 29.79 & 33.41 & 2779.6 & 16.03 & 380 & 62.9 & 81.90 & 16486 & 38.51 \\
\hline 180 & 31.94 & 37.04 & 3396.8 & 18.17 & & {$[62.5]$} & {$[74.98]$} & [14226] & {$[37.54]$} \\
\hline 200 & 34.25 & 40.52 & 4058.4 & 20.23 & 390 & 67.6 & 83.59 & 17136 & 39.65 \\
\hline 220 & 36.82 & 43.91 & 4768.7 & 22.23 & & {$[67.4]$} & {$[76.67]$} & [14876] & {$[38.53]$} \\
\hline 240 & 39.68 & 47.23 & 5533 & 24.17 & $396.3^{b}$ & $(>300)$ & $(84.69)$ & (17570) & $(40.36)$ \\
\hline 260 & 42.99 & 50.53 & 6359 & 26.07 & & [70.45] & {$[77.77]$} & [15310] & [39.14] \\
\hline 280 & 47.22 & 53.86 & 7259 & 27.94 & \multirow{2}{*}{\multicolumn{5}{|c|}{ Mesomorphic phase }} \\
\hline \multirow[t]{2}{*}{$290^{a}$} & 49.87 & 55.57 & 7744.0 & 28.86 & & & & & \\
\hline & {$[49.87]$} & {$[55.57]$} & {$[7744.0]$} & {$[28.86]$} & $396.3^{\circ}$ & $(>300)$ & $(86$ & $(183$ & $(40.36)$ \\
\hline \multirow[t]{2}{*}{298.15} & 52.71 & 56.99 & 8161.0 & 29.62 & & {$[80.10]$} & {$[77.77]$} & {$[15310]$} & [39.14] \\
\hline & {$[51.90]$} & {$[56.98]$} & {$[8158.4]$} & {$[29.62]$} & 400 & $(>150)$ & $(87.34)$ & $(18621)$ & $(40.79)$ \\
\hline \multirow[t]{2}{*}{300} & 53.20 & 57.30 & 8254.7 & 29.78 & & {$[80.43]$} & {$[78.52]$} & {$[15607]$} & {$[39.50]$} \\
\hline & {$[52.30]$} & {$[57.30]$} & {$[8254.7]$} & {$[29.78]$} & 410 & 81.4 & 89.34 & 19430 & 41.95 \\
\hline \multirow[t]{2}{*}{310} & 58.00 & 59.06 & 8790.0 & 30.71 & & [81.4] & {$[80.52]$} & [16416] & {$[40.48]$} \\
\hline & {$[54.76]$} & {$[59.06]$} & {$[8790.0]$} & {$[30,71]$} & 420 & 82.3 & 91.31 & 20248 & 43.10 \\
\hline \multicolumn{2}{|c|}{$318.5^{b}(\approx 10500)$} & $(60.57)$ & (9264.3) & (31.48) & 430 & 82.7 & 93.25 & 21073 & 44.24 \\
\hline \multirow{3}{*}{\multicolumn{2}{|c|}{$[56.84]$}} & {$[60.57]$} & [9264.3] & [31.48] & 440 & 83.1 & 95.16 & 21902 & 45.38 \\
\hline & & Phase II & & & $\begin{array}{l}450 \\
4607^{b}\end{array}$ & $\begin{array}{c}83.8 \\
2000\end{array}$ & $\begin{array}{l}97.04 \\
(0880)\end{array}$ & 22737 & 46.51 \\
\hline & & $(66.57)$ & $(11176.2)$ & $(31.48)$ & & $\begin{array}{l}(>200) \\
{[84.5]}\end{array}$ & $\begin{array}{l}(98.89) \\
{[90.13]}\end{array}$ & $\begin{array}{l}(23580) \\
{[20595]}\end{array}$ & $\begin{array}{l}(41.11) \\
45.43\end{array}$ \\
\hline \multirow{2}{*}{\multicolumn{2}{|c|}{$\begin{aligned} 318.5^{b}(\approx 10500) \\
{[56.84] }\end{aligned}$}} & {$[60.57]$} & {$[9264.3]$} & [31.48] & \multicolumn{5}{|c|}{ Isotropic liquid } \\
\hline & & & & & $460.7^{b}$ & $(>200)$ & (99.33) & (23781) & $(47.71)$ \\
\hline
\end{tabular}

${ }^{a}$ Quantities in square brackets represent estimated lattice heat capacities at indicated temperature.

${ }^{b}$ Quantities in parentheses represent estimated heat capacities or other thermodynamic functions estimated on the assumption that the transitions are truly isothermal at the transition temperatures indicated. 
TABLE 7. Summary of transition temperatures for thallium(I) $n$-tetradccanoate

\begin{tabular}{lcccc}
\hline \multicolumn{1}{c}{ Phases } & $\begin{array}{c}\text { This work } \\
\text { (adiabatic } \\
\text { calorimetry) }\end{array}$ & $\begin{array}{c}\text { This work } \\
\text { (d.s.c.) }\end{array}$ & $\begin{array}{c}\text { Lindau } \text { et al. } .^{(7.8)} \\
\text { (d.s.c.) }\end{array}$ & $\begin{array}{c}\text { Meisel } \text { et al. } .^{(3)} \\
\text { (d.t.a.) }\end{array}$ \\
\hline IV $\rightarrow$ III & - & - & 309.7 & - \\
III $\rightarrow$ II & 318.5 & 318.2 & 315.2 & 313 \\
II $\rightarrow$ I & - & 378.0 & 368.0 & 371 \\
I $\rightarrow$ Mesophase & - & 396.3 & 392.1 & 393 \\
Mesophase $\rightarrow$ Isotropic liquid & - & 460.7 & 457.6 & 460 \\
\hline
\end{tabular}

as low as $12 \mathrm{~K}$. The odd-even effect noted previously with the lighter alkali metal does not obtain since it is overwhelmed by the massiveness of the thallium atoms.

\section{REFERENCES}

1. Boerio-Goates, J.; Lopez de la Fuente, F. L.; Cheda, J. A. R.; Westrum, E. F., Jr. J. Chem. Thermodynamics 1985, 17, 401.

2. Ngeyi, S. P.; Lopez de la Fuente, F. L.; Cheda, J. A. R.; Fernandez-Martin, F.; Westrum, E. F., Jr. J. Chem. Thermodynamics 1985, 17, 409.

3. Meisel, T.; Seybold, K.; Halmos, Z.; Roth, J.; Melykuti, Cs. J. Therm. Anal. 1976, 10, 419.

4. Westrum, E. F., Jr.; McCullough, J. P. Experimental Thermodynamics. Scott, D. W.; McCullough, J. P.: editors. Butterworths: New York. 1968, p. 337.

5. Westrum, E. F., Jr. Proceedings NATO Advanced Study Institute on Thermochemistry at Viana do Castello, Portugal. Ribeiro da Silva, M. A. V.: editor. Reidel: New York. 1984, 745.

6. Gallot, B.; Skoulios, A. Kolloid-Z. 1966, 209, 164.

7. Lindau, J.; Diele, S.; Krüger, H.; Dörfler, H.-D. Z. Phys. Chemie Leipzig 1981, $262,775$.

8. Lindau, J.; König, H.-J.; Dörfler, H.-D. Colloid and Polymer Science 1983, 261, 236.

9. Ngeyi, S. P.; Westrum, E. F., Jr.; Franzosini, P. J. Chem. Thermodynamics 1986, 18, 609.

10. Franzosini, P.; Ngeyi, S. P.; Westrum, E. F., Jr. J. Chem. Thermodynamics 1986, 18, 1169

11. Ngeyi, S. P.; Westrum, E. F., Jr.; Franzosini, P. J. Chem. Thermodynamics 1987, 19, 121. 